\title{
Erratum to: Mortality prediction after cardiac surgery: blood lactate is indispensible. Thorac Cardiovasc Surg 2012 (epub ahead of print)
}

\author{
Akmal M. A. Badreldin ${ }^{1,2}$ Bernhard R. Brehm ${ }^{1}$ Sherif Elsobky ${ }^{3}$ Mohamed Abul-dahab ${ }^{4}$
}

Thomas Lehmann $^{5}$ Ole Bayer $^{6}$ Thorsten Wahlers ${ }^{7}$ Khosro Hekmat ${ }^{1,7}$

${ }^{1}$ Cardiothoracic Surgery, CardioClinic, Cologne, NRW, Germany

${ }^{2}$ Department of Cardiology, Catholic Hospital Koblenz-Marienhof, Koblenz, Germany

3 Wolfson Medical School, University of Glasgow, University Avenue,

Address for correspondence Akmal M. A. Badreldin, MD, OscarAbisch-Weg 34, 51145 Cologne, Germany

Glasgow, United Kingdom

${ }^{4}$ Department of Cardiothoracic Surgery, Kasr-El-Aini School of

Medicine, Cairo University, Egypt

${ }^{5}$ Department of Medical Statistics, Computer Sciences and

Documentation, Friedrich-Schiller-University of Jena, Jena, Germany

6 Department of Anesthesiology and Intensive Care Medicine,

Friedrich-Schiller-University of Jena, Jena, Germany

7 Department of Cardiothoracic Surgery, University of Cologne,

Cologne, Germany

Thorac Cardiovasc Surg 2013;61:375-376.

\section{ERRATUM}

It has been brought to the Editor-in-Chief's attention on March 18, 2013 that there is a mistake in the list of authors of the following publication, published eFirst on March 11, 2013. DOI: http://dx.doi.org/10.1055/s-0032-1324796.

The correct order of authors and their affiliations is as follows:

Akmal M. A. Badreldin ${ }^{1,2}$ Fabian Doerr ${ }^{3}$ Bernhard R. Brehm ${ }^{2}$ Mohamed Abul-dahab ${ }^{4}$ Thomas Lehmann ${ }^{5}$ Ole Bayer ${ }^{6}$ Thorsten Wahlers ${ }^{7}$ Khosro Hekmat ${ }^{1,7}$

${ }^{1}$ Cardiothoracic Surgery, CardioClinic, Cologne, NRW, Germany

${ }^{2}$ Department of Cardiology, Catholic Hospital Koblenz-Marienhof, Koblenz, Germany

${ }^{3}$ Faculty of Medicine, Friedrich-Schiller-University of Jena, Erlanger Allee 101, Jena, Germany ${ }^{4}$ Department of Cardiothoracic Surgery, Kasr-El-Aini School of Medicine, Cairo University, Egypt

${ }^{5}$ Department of Medical Statistics, Computer Sciences and Documentation, FriedrichSchiller-University of Jena, Jena, Germany

${ }^{6}$ Department of Anesthesiology and Intensive Care Medicine, Friedrich-Schiller-University of Jena, Jena, Germany

${ }^{7}$ Department of Cardiothoracic Surgery, University of Cologne, Cologne, Germany

Markus K. Heinemann, MD, PhD Editor-in-Chief

The Thoracic and Cardiovascular Surgeon 